\title{
Link Sense: Beyond Wireless Carrier Sensing
}

\author{
Naveen Santhapuri, Member, IEEE, Romit Roy Choudhury, Member, IEEE, and Srihari Nelakuditi, Member, IEEE
}

\begin{abstract}
Carrier sensing is the act of listening on a wireless channel before initiating a transmission. It is intrinsic to modern random access protocols to avoid collisions. However, carrier sense is only a binary indicator of whether the channel is idle or busy. Knowing why it is busy (i.e., which senderreceiver pair is currently communicating) would be very useful in scheduling concurrent transmissions. While this information could be decoded from the frame header, it is not always feasible especially when the listener is outside the decoding (but within the sensing) range. Even if the listener is within decoding range, it may not be able to decode the header because of interference, or perhaps because it was sleeping. We propose LinkSense that leverages OFDM subcarriers to embed a per-link signature in each transmission. With LinkSense, a listener can decide to sense the channel at any given time, and identify which links are active in the vicinity. The listener need not be within decoding range of the active links, and can sense the channel only for a few OFDM symbol durations. We explore the feasibility of LinkSense and implement/validate it using USRPs/GNU Radios.
\end{abstract}

Index Terms-Wireless channel access, carrier sense.

\section{INTRODUCTION AND MOTIVATION}

W IRELESS medium access protocols like 802.11 perform physical carrier sensing (CSMA/CA). Under CSMA/CA, a transmitter senses the wireless channel and initiates a transmission only if there is no activity sensed. While this is helpful in avoiding collisions, it is a conservative mechanism which reduces spatial reuse. The key underlying assumption is that simultaneous transmissions by two nodes that are in the carrier sense range will result in a packet loss due to collision. But this leads to the well-known exposed terminal problem in wireless networks. An exposed transmitter, which senses an active transmission by another nearby transmitter, defers its own transmission though they may not interfere with each other's reception [1]. Moreover, even when they cause mutual interference, the transmissions could still be successfully received albeit at lower bit rates [2]. Such opportunities for better channel usage are not allowed by carrier sensing since it is too coarse-grain an indicator of channel activity.

The information gathered from carrier sense is simple: whether there is some activity on the channel or not. It only indicates an on-going transmission but does not reveal who is transmitting to who. Additional information like the identity of the transmitter and the receiver will aid the MAC layer to

Manuscript received November 11, 2010. The associate editor coordinating the review of this letter and approving it for publication was D. Michalopoulos.

N. Santhapuri and R. Roy Choudhury are with the Department of Electrical and Computer Engineering, Duke University, Durham, NC 27708 (email: naveen.santhapuri@duke.edu, romit@ee.duke.edu).

S. Nelakuditi is with the Dept. of Computer Science and Engineering, University of South Carolina, Columbia, SC 29208 (e-mail: srihari@ cse.sc.edu).

Digital Object Identifier 10.1109/LCOMM.2011.020111.102197 make better decisions and potentially improve spatial reuse. The knowledge of active links in the neighborhood can be helpful in detecting exposed terminals. Concurrent transmission schemes like CMAP [1] initiate transmissions based on such knowledge. Rate control schemes can also benefit from knowing the presence of interferers by adjusting the rate based on the interference caused by the active transmitters. Knowing who is active will also be helpful in diagnosing whether a packet loss is because of fading or collision.

There are several existing ways to glean this additional information from the MAC layer. Active link information can be obtained by listening to the RTS/CTS control frames in 802.11 which are exchanged before the DATA frame. But RTS/CTS mechanism is turned off by default in most installations as it is observed to decrease overall throughput [3]. Partially decoding the frame header while overhearing the transmission will also help in determining the sender and receiver of the communication. However, it is not always possible to overhear/decode the headers, especially when the listener is outside the communication range (though inside the sensing range) of the active transmitter. Moreover, if a listener fails to overhear/decode the header for any reason, it would not know of the active link for the entire duration of the packet.

As an alternative, we propose an Orthogonal Frequency Division Multiplexing (OFDM) based physical layer approach for sensing active links. Currently, an 802.11 transmitter using carrier sensing, measures the energy on the entire frequency spectrum corresponding to the channel, and uses a cut-off threshold to determine if there is activity. Logically, this (activity/inactivity) is one bit of information. A similar but fine-grained approach can make it possible to detect if there is activity on a specific subcarrier. The activity (energy) on a subcarrier can be controlled at the transmitter. In other words, a bit of information can be obtained from one subcarrier as opposed to the entire spectrum. This subcarrier sensing can be used in many contexts. But in this work, we employ it for sensing active links. If a small subset of the subcarriers (say $m$ ) is used for this purpose, it is possible to transmit upto $m$ bits of information about the link by activating/deactivating the subcarriers. Such a sequence of $m$ bits can serve as a signature for a link, if that sequence can be uniquely mapped to that link. We call this approach LinkSense.

There are several benefits with the LinkSense approach. By using a small subset of subcarriers to transmit a signature, an active link can be detected anytime during the packet duration. It is not necessary to overhear/decode the MAC header as each OFDM symbol carries the signature. This also leads to faster detection of the active link. It is also possible for a node to sense active links outside its communication (but inside its sensing) range. We believe this is a proactive but natural 
extension, to the traditional physical carrier sensing, to obtain more information about the channel status.

The rest of the paper is organized as follows. The next section presents the related work. Section III describes LinkSense in detail. Section IV demonstrates LinkSense with implementation on USRP/GnuRadio. Section V concludes the paper.

\section{RELATED WORK}

The idea of making use of transmitter and receiver identity to improve concurrency has been explored earlier [1]. They use RTS/CTS, pilot packet, or additional information after the MAC header for conveying active link. These schemes, however, assume that a header/pilot packet can always be overheard/decoded which, as we explained before, may not always be feasible. In [4], the authors propose a radiometric signature scheme for detecting the transmitter by taking advantage of the unique physical makeup of each wireless transmitter. A similar approach based on signal reception signatures is taken for location distinction in [5]. These two works identify only the transmitter, not the receiver (link). Recently, it has been shown that OFDM subcarriers can be used to get simultaneous multiple acknowledgments for a multicast packet [6]. This approach is similar to ours in the assignment phase but the purpose of our scheme is to detect active links.

\section{LINKSENSE}

In this section, we first provide a brief background on OFDM subcarriers. Then we present the intuition behind LinkSense and discuss the challenges in implementing it.

\section{A. Background}

Orthogonal Frequency Division Multiplexing (OFDM) is a physical layer technique used in $802.11 \mathrm{a} / \mathrm{g}$ networks. OFDM uses multiple closely spaced small bandwidth carriers called subcarriers, which are orthogonal and hence non-interfering. Each individual sub-carrier contains modulated data symbols and a separate modulation scheme can be used on each subcarrier. The information transmitted on all the sub-carriers in one step is called an OFDM symbol. There are many subcarriers in an OFDM symbol (54 for $802.11 \mathrm{a} / \mathrm{g}, 256$ for 802.16 and even higher for ultra wide band technologies). If we select a small subset of these subcarriers (say 10\%) and activate/deactivate them, the subset will look like a bit map. Suppose, among subcarriers numbered 1 to 4, if we turn off 2 and turn on 1, 3, and 4, we can see a bitmap 1011 for these subcarriers. We use this capability for link sensing.

\section{B. Our Approach}

A node uses physical carrier sensing to know if any node in the vicinity is currently transmitting, by detecting the energy on the channel. Our aim is to employ such energy detection but also sense the active link, i.e, the sender-receiver pair of the transmission. The received signal samples values, before any processing, are shown in Fig. 1. These figures correspond to In-phase (I) and Quadrature (Q) values of $<0,0\rangle$ and $\langle 2,2\rangle$ in the constellation space. The $<0,0\rangle$ samples have low energy compared to the $<2,2>$ samples and a proper energy thresholding (similar to carrier sense) can

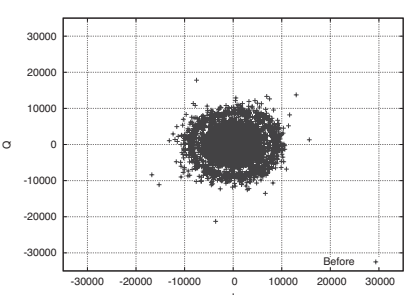

(a) $\mathrm{I}, \mathrm{Q}=(0,0)$

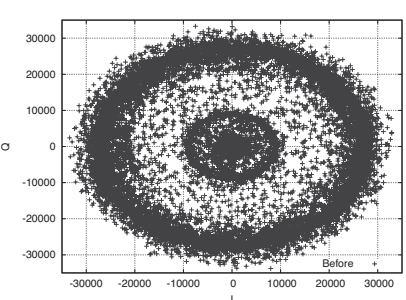

(b) $\mathrm{I}, \mathrm{Q}=(2,2)$
Fig. 1. Constellation diagrams before correlation/equalization at the receiver. An inactive subcarrier (symbol $<0,0\rangle$ ) can be distinguished from an active one (symbol $<2,2>$ ) with proper energy thresholding, similar to carrier sense.

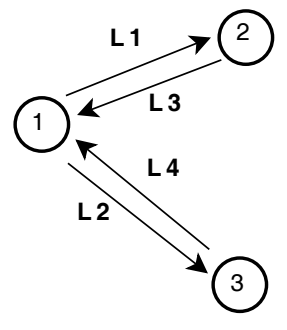

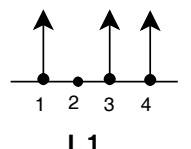

L1

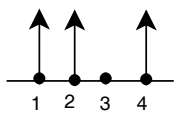

L3
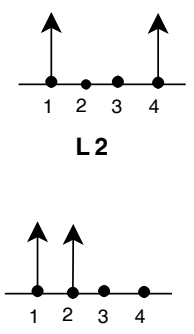

L 4
Fig. 2. Subcarrier maps which serve as signatures for 4 links.

help detect whether a subcarrier is active or not. We make use of this capability and propose LinkSense. LinkSense employs intentional loading of a small set of OFDM subcarriers with a predetermined carrier bit map, where a bit value one indicates an active subcarrier and zero indicates an inactive subcarrier. A node using LinkSense can detect the energy on each of the subcarriers and determine which subcarriers are active and which are not. This will help the node in determining the carrier map being used by the transmitter. If this carrier map is unique for each link, this can serve as a link signature.

Consider the scenario in Fig. 2 where there are 4 links to and from node 1 . If each node activates a unique set of subcarriers for each of its outgoing links, each link will have a unique ID in the network. An example assignment of subcarriers is shown where link L1 is assigned a bit map 1011 and L2 1001, distinguishing the links coming out of node 1 . If $\mathrm{L} 1$ is active, node 3 will detect activity on subcarriers 1,3 and 4 and will be able to determine that node 1 is transmitting to node 2 .

\section{Challenges}

There are several practical challenges in realizing LinkSense. Most of them pertain to signature assignment. We discuss some of the critical challenges below.

Detecting Multiple Links: An application of LinkSense is improved concurrency in the wireless network. This also increases the probability of hearing simultaneous transmissions, when a receiver is trying to detect activity on the channel. This will need detection of multiple links simultaneously. If the signatures are chosen carefully, mulitple transmissions will result in a unique combination signature that can be used to identify the participating links. For example, when link1 is assigned 0100 and link 2 is assigned 1010 as signatures, detecting a 1110 might indicate simultaneous transmissions by link 1 and link 2 . The combined signature should result 

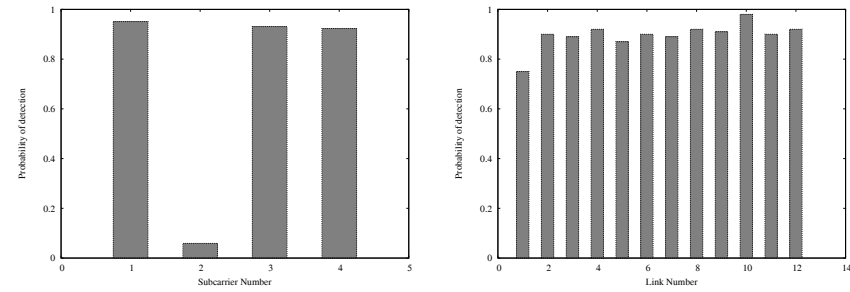

Fig. 3. Detection probability of active subcarriers and links: (a) four subcarriers corresponding to signature 1011; (b) twelve different links.

in a bitwise OR of both the signatures. Of course, there can be destructive interference, but careful thresholding and redundancy in the signature bits can eliminate this problem. Initiating a transmission when more than 3 links are active may not be feasible and hence detection of more than 2 simultaneous links may not be necessary.

Signature Overhead: The purpose of assigning a signature to a link and subsequent detection of active links is to derive potential performance gain from that knowledge. It is possible that any gains in concurrency achieved by using LinkSense might be negated due to the signature overhead. Therefore, careful assignment of signatures is necessary. Fortunately, there are opportunities for reusing link signatures that do not cause any ambiguities at a sensing node. For example, the links that are not in the adjacent collision domains may be assigned the same signature. Even when they are in the same collision domain, same signature may be assigned to two links if they can be disambiguated based on the signal strength disparities at the receivers. We need to explore signature assignment strategies to ensure that LinkSense can be effective with as few subcarriers as possible for a signature.

Inter-Carrier Interference: We observed a significant amount of energy on adjacent deactivated subcarriers when a subcarrier is active. We believe this is because of a very narrow bandwidth for each subcarrier in the GNU Radio implementation on USRP. In typical 802.11 wireless channels with $20-40 \mathrm{MHz}$ bandwidth, the spacing between subcarriers is much higher. As a result, the intercarrier interference can be minimized even when using adjacent subcarriers. We expect the detection probability to increase significantly with higher bandwidth channels. In our implementation, we use every 3rd subcarrier (making the ones in between zero) for link signature. In the next section, we discuss the implementation details of LinkSense and the present the evaluation results.

\section{IMPLEMENTATION AND EVALUATION}

We use the GNU Radio/USRP platform for evaluating LinkSense. The OFDM implementation in GNU Radio is still not well developed and hence can operate only at very low bandwidth $(500 \mathrm{KHz})$. We used a set of 64 subcarriers, with an FFT size 64 and BPSK modulation. We implemented a proof of concept for LinkSense by using this OFDM implementation. In each of our experiment runs, the transmitter transmits 1000 packets of data, each of size 512 bytes, continuously to the receiver. All USRPs operate on $2.43 \mathrm{GHz}$ as center frequency. All the experiments presented were conducted in a university lab with many wireless access points and clients active in the $2.43 \mathrm{GHz}$ range. Below we present the results for detecting the energy on subcarriers and sensing active links.

Detecting Active Subcarriers: In this experiment, we have one USRP node transmitting to a receiver and a passive listener detects the active subcarriers among the ones assigned for LinkSense. We use 4 subcarriers for signature. The transmitter activates subcarriers 1, 3, and 4 (signature 1011). The receiver counts the number of symbols in which each subcarrier is detected as active. Fig. 3a shows the results. All the active subcarriers were detected in more than $90 \%$ of the received symbols. But there were a few false positives (about 5\%) for subcarrier 2. This result shows that the active subcarriers can be detected with a very high probability.

Detecting Active Links: Once we established the feasibility of detecting active subcarriers, the next step is to try and detect active links. In this experiment, we use 5 USRPs with one of them acting as a passive receiver and the other 4 acting as transceivers. With 4 active nodes, we have 12 links. We assign 12 codes (1 through 12) for each of the directional links. Each node uses 4 subcarriers for transmitting the signature and the rest for transmitting data. All the transmitters and receivers are tuned to the $2.43 \mathrm{GHz}$ frequency and sequentially transmit data on each of their links. The receiver detects the signature transmitted when each link alone is active. The signature detected on each symbol is recorded and the probability of detecting the transmitted signature is plotted in Fig. 3b (each data point is based on at least 15000 symbols). It is evident that the unique signature of each link can be detected with high accuracy. The differences in link detection accuracy is due to occasional false positives in detecting some active sucarriers. Our evaluation, though preliminary, demonstrates the feasibility of LinkSense on a small testbed.

\section{Conclusions}

We argued that carrier sensing is not fine-grain enough indicator of wireless channel activity to facilitate concurrent transmissions. We proposed LinkSense based on utilizing a few OFDM subcarriers for conveying the link signature in each symbol, enabling sensing of active links at any instant. We demonstrated the feasibility of LinkSense on a small USRP testbed. While the results are promising, we need to address several challenges and investigate various tradeoffs w.r.t. signature assignment for LinkSense to be practically beneficial.

\section{REFERENCES}

[1] M. Vutukuru, K. Jamieson, and H. Balakrishnan, "Harnessing exposed terminals in wireless networks," in NSDI, 2008.

[2] J. Lee and et al., "An experimental study on the capture effect in 802.11a networks," in WinTech, 2007.

[3] J. Li, C. Blake, D. S. J. D. Couto, H. I. Lee, and R. Morris, "Capacity of ad hoc wireless networks," in Mobicom, 2001.

[4] V. Brik, S. Banerjee, M. Gruteser, and S. Oh, "Wireless device identification with radiometric signatures," in Mobicom, 2008.

[5] J. Zhang, M. Firooz, N. Patwari, and S. Kasera, "Advancing wireless link signatures for location distinction," in Mobicom, 2008.

[6] A. Datta and et al., "SMACK-a SMart ACKnowledgment scheme for broadcast messages in wireless networks," in SIGCOMM, 2008. 